hospital, particularly its role in managing patients concerns and avoiding hospital admission.

Methods A retrospective audit of 1000 IBD patient calls between August 2019 and October 2019 was conducted. The help line covered patients across all Imperial Trust sites - Charing Cross, St Mary's and Hammersmith Hospitals. The data collected included the following - Time of call, diagnosis, mode of contact, when the call was answered, number of attempts, advice given by whom, call reason and outcome.

Results The divide between UC and Crohn's was approximately equal $(46.3 \%$ vs $48.5 \%)$. Out of the 1000 the majority (809) were direct patient contact. Voicemail was the most common mode of contact (577) followed closely by email (435).

$84 \%$ of patients were answered on the same day, $12 \%$ the next day and $4 \%$ on another day. $84 \%$ of patients got through on the first attempt while $11 \%$ needed a second attempt and $5 \%$ required three attempts. Majority of the advice was given via telephone $(70 \%)$, the second most common was email (24\%).

Most calls (25\%) were regarding investigation/treatment, $21 \%$ regarding flares, $12 \%$ results, $11 \%$ admin, $11 \%$ advice, $7 \%$ homecare, $4 \%$ earlier appointment and 4\% side effects.

Regarding outcomes - $28 \%$ involved investigation/treatment, $13 \%$ repeat prescriptions, $13 \%$ results, $12 \%$ advice, $7 \%$ appointments, 5\% admin, 3\% dose escalation, 3\% home care, $3 \%$ contacted the consultant, $2 \%$ biologics switch, $2 \%$ A\&E/ urgent care referrals and $1 \%$ support.

Conclusions The service was highly efficient; $85 \%$ of calls were answered $<24$ hours while the clear majority could get through on the first attempt. A significant amount of patient contact was via email - allowing flexibility of contact between IBD Specialist nurses, patients and medical team.

The majority of calls and outcomes related to investigation, treatment and disease flare-up - demonstrating that the service is being used appropriately.

Only $2 \%$ of patients required A\&E/urgent care referrals, demonstrating that access to specialist advice can reduce or avoid costly hospital admissions. These data are in keeping with systematic reviews that have all shown advice lines to be safe and cost-effective. Medication advice and monitoring was a common use of the advice line - this remote service helps provide a robust platform for toxicity surveillance.

In order to maintain the high quality of the service, ongoing IBD Nurse education and prescribing, can help maintain high levels of efficiency, good patient care and a high level of patient satisfaction.

\section{P369 IMPLEMENTING A BIOLOGIC SWITCH PATHWAY TO FACILITATE TRANSITION TO BIOSIMILAR}

${ }^{1}$ Francesca Mastaglio*, ${ }^{2}$ Barbara Robertson, ${ }^{3}$ Diane Crake, ${ }^{4}$ Norma McConnell, ${ }^{5}$ Nicola Brownlee, ${ }^{1}$ Julie Fyall, ${ }^{1}$ Max Groome, ${ }^{1}$ John Todd, ${ }^{1}$ Jacqueline Paterson, ${ }^{1}$ Craig Mowat. 'Gastroenterology, NHS Tayside, Dundee, UK; ${ }^{2}$ Outpatents, NHS Tayside, Dundee, UK; ${ }^{3}$ Rheumatology, NHS Tayside, Dundee, UK; ${ }^{4}$ Administration and Clerical Service, NHS Tayside, Dundee, UK; ${ }^{5}$ Pharmacy, NHS Tayside, Dundee, UK

\subsection{6/gutjnl-2020-bsgcampus.443}

Introduction Biosimilar switches are likely to become a common scenario across specialties, can deliver significant savings, but can present a logistical dilemma. We implemented a Biologic Switch pathway focused on group Patient Education
Sessions (PES), devised by rheumatology colleagues, to facilitate a rapid patient-centred switch to biosimilar adalimumab in our IBD service.

Methods Adalimumab users were identified from IBD service excel spreadsheet of recorded users, Lloyds Pharmacy dispensing records and review of Clinic letters. Clerical support set up a 1 hour PES 'clinics' on the hospital patient management system (Trakcare); 10 patients per group session. Each patient was asked to record their preferences in an information letter explaining the switch and an optional invitation to attend the PES. A Registered General Nurse with no specialist knowledge of IBD was seconded for 10 weeks as a Biologics Switch nurse to deliver PES. On attendance, patients could ask the Biologic Switch Nurse questions about biosimilar, received pen device demonstration and tuition, a copy of manufacturer's booklet, Biologics Alert Card.

Results 138 patients were identified of which 132 were verified as receiving Humira and invited to attend a PES. 32 (24\%) patients were happy to switch without PES, 43 (33\%) patients accepted PES appointment but did not attend. 14 PES were delivered over 6 weeks.

Groups were observed to discuss individual experiences of their IBD, and how adalimumab had changed their lives for the better. Their main concerns were that the biosimilar might be less effective and whether they could they 'switch' back in that scenario.

Those patients happy to switch without attending PES and those who Did Not Attend PES were called by the Nurse to confirm current frequency and device used.

$125 / 132$ patients completed the switch. New prescriptions were completed, a database of biosimilar patients was set up, the old database was archived, and GP letters were dictated.

7 patients did not switch: 2 discontinued biologic (remission) 2 had surgical resection 2 switched to an alternative biologic and one refused to switch on principle and remains on Humira.

Conclusions PES can facilitate biosimilar switch in a rapid patient-centred fashion. Many patients are happy to switch without attendance. Extending the notice period may improve attendance of patients who would like to attend. PES could be extended to biologic 'new starts' too.

\section{P370 ACCEPTABILITY AND OUTCOME OF INVESTIGATING IRON DEFICIENCY ANAEMIA IN THE ELDERLY}

Alec Maynard*, Jessica Johnson, John Hebden, Stuart Riley, David Dacosta. Sheffield Teaching Hospitals NHS Foundation Trust, Sheffield, UK

\subsection{6/gutjnl-2020-bsgcampus.444}

Introduction The investigation of iron deficiency anaemia (IDA) in the over 80-year-olds presents a unique challenge due to co-morbidity, uncertain prognosis and differing attitudes to acceptability of invasive investigation. We evaluated the investigations accepted and the subsequent outcomes for elderly patients.

Methods We prospectively collected consecutive GP referrals of IDA patients aged $80+$ between 2015 and 2018 seen by a single gastroenterologist. The options of invasive investigation (bidirectional endoscopy), non-invasive investigation (CTC CT pneumocolon; CT long oral prep; CT TAP - CT thorax, abdomen, pelvis), or no investigation, were discussed and offered in a standardised manner. 


\begin{tabular}{llll}
\multicolumn{2}{l}{ Abstract P370 Table 1} & & \\
\hline AGE GROUP n=number & $\begin{array}{l}80-84 \\
(\mathbf{n}=99)\end{array}$ & $\begin{array}{l}85+ \\
(\mathbf{n}=74)\end{array}$ & $\begin{array}{l}\chi^{2} \text { comparison } \\
\text { of age group }\end{array}$ \\
\hline Declined all investigations & $9(9 \%)$ & $19(26 \%)$ & $\mathrm{P}<0.01$ \\
Bidirectional endoscopy & $37(37 \%)$ & $2(3 \%)$ & $\mathrm{P}<0.00001$ \\
Colonoscopy & $38(38 \%)$ & $2(3 \%)$ & $\mathrm{P}<0.00001$ \\
OGD & $62(63 \%)$ & $31(42 \%)$ & $\mathrm{P}<0.01$ \\
CTC & $28(28 \%)$ & $24(32 \%)$ & $\mathrm{P}=0.55$ \\
CT long oral prep & $21(21 \%)$ & $26(35 \%)$ & $\mathrm{P}<0.05$ \\
CT TAP & $13(13 \%)$ & $20(27 \%)$ & $\mathrm{P}<0.03$ \\
\hline
\end{tabular}

Results 173 patients (64.7\% female, 80-95 yrs) were seen. $28(16.2 \%)$ declined all investigations, while 39 (22.5\%) underwent bidirectional endoscopy. Investigations accepted and performed were: OGD 93 (53.7\%), colonoscopy 40 (23.1\%), CTC 52 (30\%), CT long oral prep 47 (27.2\%), and CT TAP 34 (19.7\%). Carcinoma was identified in 21 (14.5\%) patients who underwent investigations - 19 (13.1\%) were gastrointestinal (GI) cancers (16 colorectal, 3 gastric), one metastatic. Two patients with GI malignancy were also diagnosed with myeloma. The 2 non-GI cancers were metastatic renal cell carcinoma and hepatocellular carcinoma. There were significant differences in investigations undertaken between the elderly (80-84 yrs) and very elderly (85 + yrs) as shown in the table 1 .

Of the 17 potentially operable GI cancers, 9 procedures with curative intent were performed ( 8 hemicolectomy, 1 transanal resection). Two of these were in patients aged $85+$. 1 subject, aged 89, was offered curative surgery but declined. Outcomes from surgery were very positive, with $8 / 9$ patients undergoing surgery still alive (median follow up 984 days, range 454-2151). The single death occurred 1247 days postclinic. This compared with on-going survival of $2 / 8$ patients who did not undergo surgery (421 and 701 days), with a median survival of 495 days (range 93-1562) for those who died.

Conclusions The over 80-year-olds with IDA are a heterogeneous group. Overall $16 \%$ of patients declined all investigations, though this rose to $26 \%$ in those aged over 85 . Non-invasive tests were much more likely to be undertaken in the $85+$ age group, with only $3 \%$ having 'gold standard' bidirectional endoscopy. Half of patients with potentially curable GI cancers discovered underwent surgery, with $8 / 9$ alive at a mean follow-up of 2.5 years.

\section{P371 AN EVALUATION OF THE IMPACT OF A VIRTUAL BIOLOGICS CLINIC ON SURROGATE BIOMARKERS IN IBD}

${ }^{1}$ Lynne Merchant* ${ }^{*}{ }^{1}$ Katherine Davidson, ${ }^{1}$ Caroline Souter, ${ }^{2}$ Charles Morecroft, ${ }^{3}$ lan Arnott. ${ }^{1}$ Pharmacy, NHS Lothian, Edinburgh, UK; ${ }^{2}$ Liverpool John Moores University, Liverpool, UK; ${ }^{3}$ The Edinburgh IBD Unit, Edinburgh, UK

\subsection{6/gutjnl-2020-bsgcampus.445}

Introduction A Virtual Biologics Clinic (VBC) was set up in a tertiary IBD unit to improve the safe and effective prescribing of adalimumab. This new care model supplemented standard of care by proactively reviewing and monitoring patients prescribed adalimumab. Decisions were made in the VBC following virtual review of the patient's IBD surrogate biomarkers in their electronic record, with the team making interventions to optimise the efficacy and safety of ongoing treatment.

Methods In a retrospective review, patients who met the project inclusion criteria were identified from the VBC clinic lists and data was collected for a 52 week period.

Figure 1 describes the data collection process.

Results A total of 127 patients were included. There was improvement in the surrogate biomarkers in the post-review period. The percentage of recorded FCP levels indicating disease remission $(<200 \mu \mathrm{g} / \mathrm{g})$ increased from $28.8 \%$ prior to $\mathrm{VBC}$ review, to $36.0 \%$ following review. Patients recording optimal trough levels $(>8.5 \mathrm{mg} / \mathrm{L})$ at the end of the data collection also improved (16.5\% vs $39.0 \%)$.

Patient participation in completing or updating the data set for the virtual reviews was promoted through distribution of monitoring packs by the VBC team (FCP, $p=0.036$ and levels, $p=0.02$ ). Requesting additional monitoring to be completed was the most common intervention made (61.0\%), followed by requests for review in the out-patient setting (24.4\%). The safety and efficacy of continuing adalimumab prescribing was reviewed by the $\mathrm{VBC}$ for nine patients who had been lost to routine follow up by standard of care.

From the 167 VBC reviews captured, ten resulted in treatment optimisation through dose escalation. These patients were then more closely managed with more frequent VBC review, an average of 1.6 times post-escalation. Nine prescriptions $(7.0 \%)$ were discontinued in the study period due to lack of evidence for safety or efficacy.

Conclusions In addition to standard of care, the VBC was shown to support the safe and effective prescribing of adalimumab. It has now been established as a model of care.

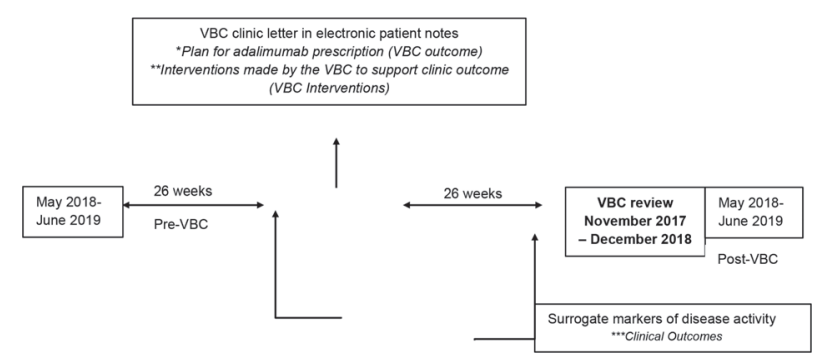

Abstract P371 Figure 1 Project design for data collection *VBC outcome:

1) Continue adalimumab prescription

2) Change dose of adalimumab

3) Stop adalimumab

4) Non-compliance monitoring letter sent to patient to encourage uptake of essential monitoring otherwise cessation of adalimumab prescription

**VBC Interventions

1) Monitoring pack sent

2) Non-compliance letter sent

3) Out-patient appointment (OPA) booked

4) OPA expedited

$* * *$ Clinical Outcomes

1) Median faecal calprotectin (FCP), C-reactive protein (CRP), adalimumab trough levels

2) Number of attended OPAs and VBCs

3) Number of IBD-related hospital admissions

4) Number of OPAs rescheduled or not attended 